\title{
Análisis comparativo: las pruebas de acceso a los estudios superiores de batería de jazz en España
}

\author{
Borja Bermúdez-Cañete ${ }^{1}$ \\ Artículo de investigación. Recibido: 11/11/2020. Aceptado: 04/02/2021. Publicación avanzada: 17/03/2021. Publicado: 01/07/2021
}

\begin{abstract}
Resumen
\end{abstract}
INTRODUCCIÓN. En España los aspirantes a cursar Estudios Superiores de Jazz se preparan para las pruebas de acceso mayoritariamente en escuelas o clases privadas de música, ya que -salvo excepciones- no es posible prepararse para ello en el sistema público de enseñanza. Dichas pruebas y sus sistemas de puntuación varían entre unos centros y otros, en algunos casos de forma drástica, lo que hace que los aspirantes tengan una doble dificultad a la hora de prepararse, especialmente los que quieren realizar la prueba en dos o más centros.

MÉTODO y RESULTADOS. Tras un análisis comparativo de todas las pruebas de acceso a los Estudios Superiores de batería de jazz de España, se han evidenciado las diferencias que existen entre ellas, y se han remarcado las similitudes para crear una "prueba tipo" que ayude a entender cómo funciona el acceso a dichos estudios en este país.

DISCUSIÓN. Además, el estudio pretende servir de ayuda a futuros aspirantes a los Estudios Superiores de Jazz, así como a profesores y a centros de Enseñanzas Profesionales que ofrecen el perfil jazz, e incluso a las autoridades encargadas de diseñar las normativas de las Enseñanzas Profesionales o las propias pruebas de acceso mencionadas anteriormente.

\section{Palabras clave}

Educación musical; Escuela de música; Música; Sistema educativo; Jazz; Enseñanzas superiores de jazz

\section{Datos del autor}

${ }^{1}$ Universidad Politécnica de Madrid. Real Conservatorio Superior de Música de Madrid, España. Correo para la correspondencia: borjabermudez@gmail.com

\section{Referencia recomendada}

Bermúdez-Cañete, B. (2021). Análisis comparativo: las pruebas de acceso a los estudios superiores de batería de jazz en España. REIRE Revista d'Innovació i Recerca en Educació, 14(2), 1-18. https://revistes.ub.edu/index.php/REIRE/article/view/32775

(C) $2021 \mathrm{El}$ autor. Este artículo es de acceso abierto sujeto a la licencia Reconocimiento 4.0 Internacional de Creative Commons, la cual permite utilizar, distribuir y reproducir por cualquier medio sin restricciones siempre que se cite adecuadamente la obra original. Para ver una copia de esta licencia, visite https://creativecommons.org/licenses/by/4.0/ 


\section{Títol (català)}

Anàlisi comparativa: les proves d'accés als estudis superiors de bateria de jazz a Espanya

Resum

INTRODUCCIÓ. A Espanya, els aspirants a cursar els estudis superiors de jazz es preparen per a les proves d'accés majoritàriament en escoles o classes privades de música, ja que -excepte algunes excepcions- no és possible preparar-se per a aquest ensenyament en el sistema públic. Aquestes proves i els seus sistemes de puntuació varien entre uns centres $\mathrm{i}$ els altres, en alguns casos de forma dràstica, $\mathrm{i}$ això fa que els aspirants tinguin una doble dificultat a l'hora de preparar-se, especialment els que volen fer la prova en dos o més centres.

MÈTODE i RESULTATS. Després d'una anàlisi comparativa de totes les proves d'accés als estudis superiors de bateria de jazz d'Espanya, $s^{\prime}$ han evidenciat les diferències que hi ha entre aquestes proves i se $n^{\prime}$ han remarcat les similituds, per crear una «prova tipus» que ajudi a entendre com funciona l'accés a aquests estudis en aquest país.

DISCUSSIÓ. Aquest article vol servir d'ajuda a futurs aspirants a estudis superiors de jazz, així com a professors i a centres d'ensenyament professional que ofereixen el perfil de jazz, i fins i tot a les autoritats encarregades de dissenyar les normatives dels ensenyaments professionals o les mateixes proves d'accés esmentades.

Paraules clau

Educació musical; Escola de música; Música; Sistema educatiu; Jazz; Ensenyaments superiors de jazz

\section{Title (English)}

Comparative analysis: the entrance audition for jazz studies (drums) in Spain

\section{Abstract}

INTRODUCTION. In Spain, applicants for the Bachelor's degree in Jazz Studies prepare for the entrance audition mainly in private music schools or with private music teachers, since - with few exceptions - no preparatory courses for it are available in the public education system. These entrance auditions and their scoring systems vary from one centre to another, in some cases quite dramatically. This means that applicants face a double difficulty when preparing for the auditions, especially those who want to apply to two or more centres.

METHOD and RESULTS. This comparative analysis of all the entrance auditions for the Bachelor's degree in Jazz Studies (drums) in Spain highlights the differences and the similarities between them in order to create a standard audition exam that can help to understand the process of gaining admission to these studies in this country.

DISCUSSION. The study provides guidance for applicants for the Bachelor's degree in Jazz Studies and their teachers, for Precollege Music Education centres that offer preparatory courses in jazz, and for the authorities in charge of designing the regulations of Precollege Jazz Programmes and the entrance auditions mentioned above.

\section{Keywords}

Music education; Music school; Music; Education system; Jazz; Jazz studies 


\section{Introducción}

Hasta finales del siglo XX era difícil pensar en que pronto se podrían realizar estudios superiores de jazz en nuestro país ${ }^{1}$. Sin embargo, en 1992, poco después de la aprobación de la LOGSE², se ofreció un atisbo de esperanza al alumnado interesado en otras músicas que no fueran la clásica, incluyéndose las palabras "jazz" y "pop" en los contenidos establecidos en la ley para las Enseñanzas de Grado Elemental y de Grado Medio $^{3}$.

El gran salto, sin embargo, se dio tres años más tarde: el Real Decreto 617/1995 (de 21 de abril) incluyó nuevas especialidades en el Grado Superior como la del jazz, la etnomusicología, el flamenco y los instrumentos de la música tradicional y popular.

En el artículo 8 del mismo Real Decreto se regulaban las pruebas de acceso para las diferentes especialidades, requiriéndose en las de jazz la "interpretación de un programa de 30 minutos, integrado por obras y/o estudios de una dificultad apropiada a este nivel", el "análisis de una obra o fragmento, propuesto por el tribunal", y la "realización de una improvisación a partir de una secuencia armónica dada por el tribunal".

La poca concreción en dicho artículo vino acompañada de las críticas de varios aspirantes, ya que al no existir Estudios Profesionales de Jazz no existía un consenso sobre la dificultad a la que se aludía en el Real Decreto mencionado (Calderón, 2016). De este modo, los primeros conservatorios que ofrecieron los Estudios Superiores de Jazz tuvieron que diseñar sus propias pruebas de acceso, que servirían de referencia a muchos de los centros que se incorporarían después a la oferta de estos estudios, pero que variarían entre unos y otros como veremos a continuación. Las pruebas, además, serían diferentes para cada instrumento, lo que ha hecho que centremos este artículo en las pruebas de batería para facilitar el estudio comparativo, si bien es cierto que la mayor parte de las conclusiones se pueden aplicar a todos los instrumentos.

\subsection{Dos décadas de Estudios Superiores de Jazz}

En el año 2001 se empezaron a ofrecer los Estudios Superiores de Jazz en la Escuela Superior de Música de Cataluña (Esmuc), y un año más tarde en el Conservatorio Superior de Música del País Vasco (Musikene) y en el Conservatorio Superior de Navarra. En la misma década se irían sumando distintas instituciones públicas y privadas a las Enseñanzas Superiores de Jazz: el Conservatorio Superior de Música del Liceu (2003, Barcelona), el Conservatorio Superior de La Coruña (2006), el Conservatorio Superior de Música Joaquín Rodrigo de Valencia (2008), el Conservatorio Superior de Música de Canarias (2008) y el Taller de Músics de Barcelona -impartiendo enseñanzas de jazz desde 1979, a las que se les dio el carácter legal de Título Superior en 2009.

Desde el comienzo, todos estos centros se vieron fuertemente influenciados por la Berklee School of Music de Boston (EEUU), "hasta el punto de ofrecer las mismas metodologías de ésta como un criterio social de calidad en la enseñanza" (Casas, 2013, p. 23). Esto se debió principalmente a dos

\footnotetext{
${ }^{1}$ En este artículo utilizaremos la palabra "jazz" en el sentido académico del término, es decir, de las diferentes enseñanzas que tratan las músicas actuales, y que suelen tener al jazz como base debido a la tradición metodológica de estas músicas. Englobaremos, bajo el término jazz, a todos los estudios que tienen cabida bajo este nombre, como "música moderna", "músicas actuales”, etc.

${ }^{2}$ Ley Orgánica General del Sistema Educativo (LOGSE), de 3 de octubre de 1990.

${ }^{3}$ Dichos contenidos se regularon en el Real Decreto 756/1992, de 26 de junio.
} 
motivos: por una parte, el gran prestigio a nivel global del que gozaba esta escuela; por otra, el hecho de que varios de los primeros profesores que comenzaron a impartir la especialidad de Jazz en España habían estudiado en dicho centro. Posteriormente, los primeros centros que empezaron a impartir jazz en nuestro país -especialmente los del País Vasco y Cataluña- se convirtieron en referencia para las siguientes instituciones que incorporaron los Estudios Superiores de dicha especialidad.

De esta forma, en la nueva década se han sumado a impartir las especialidades relacionadas con el jazz y las músicas actuales centros como la Universidad Alfonso X el Sabio -que ofrece los estudios equivalentes de Grado en Madrid desde 2014-, el Conservatorio Superior de Música de las Islas Baleares (2015), la Escola Jam Session de Cataluña -que desde 2015 implanta una especialidad enfocada hacia el rock-, la Escuela de Música Creativa de Madrid (2016), el Conservatorio Superior de Música Manuel Castillo de Sevilla (2017), el Conservatorio Mayeusis de Vigo (2018), el Conservatorio Superior de Música de Málaga (2019) y el Centro Superior de Música de Galicia (en Valga, 2020).

\subsection{El problema de la preparación de las pruebas de acceso}

De los datos anteriores podríamos deducir que hay varias opciones para estudiar jazz en nuestro país, si bien es cierto que se observa un gran vacío en las regiones del centro peninsular, quedando como únicas opciones dos centros privados en Madrid.

Todo esto provoca que los estudiantes de varias regiones tengan que ir a centros de otras comunidades autónomas si quieren estudiar jazz. De esta forma, tal y como ocurre en los estudios de música clásica, muchos de ellos deciden prepararse para dos o más pruebas de acceso distintas, con las dificultades que esto pueda entrañar.

Además, existe un hándicap extra para los estudiantes de jazz de España: mientras que los estudiantes de interpretación de música clásica pueden formarse en la amplia red de conservatorios profesionales públicos estatales, los que aspiran a estudiar jazz preparan la prueba de acceso sobre todo con profesores particulares y en escuelas privadas. Estas últimas, según Andueza (2017), aprovechan la ausencia de oferta formativa de jazz en las instituciones públicas ofreciendo cursos propios.

Cabe señalar que, en el año 2006, con la llegada de la LOE, se implantaron las especialidades de guitarra eléctrica y bajo eléctrico en varios conservatorios profesionales, pero por ahora no son el homólogo del clásico para la preparación de las pruebas de acceso a los Estudios Superiores. Dichas especialidades se imparten desde un enfoque de la música clásica, no disponen asignaturas específicas como "combo" o armonía moderna -salvo en Cataluña-, y la asignatura de conjunto no se aprovecha al máximo, ya que otros instrumentistas no reciben formación moderna en clase de instrumento (Bernal, 2013).

Por otra parte, también en 2006 se abrió la vía para que los conservatorios profesionales implantasen el perfil jazz en los últimos dos años de sus enseñanzas, es decir, en quinto y sexto curso. Desde entonces, esta vía se oferta en ciertos conservatorios para gran parte de las especialidades clásicas; sin embargo, los estudiantes que eligen este perfil tienen que seguir estudiando las asignaturas obligatorias características de la música clásica. Además, estos continúan trabajando el repertorio 
clásico en la clase de instrumento, por lo que el itinerario jazz sólo supone una introducción a esta música, lejos de servir para alcanzar el nivel necesario para acceder a los Estudios Superiores de Jazz.

Por último, hay que añadir que en el año 2017 la Generalitat de Cataluña aprobó mediante el Decreto 5/2017 (de 17 de enero) la especialidad de percusión "de la música moderna y jazz" para los conservatorios profesionales. Aunque esto ha significado un paso más para la inclusión del jazz en el sistema público de enseñanza, que tal vez podrán seguir próximamente otras comunidades autónomas, el poco tiempo que lleva existiendo esta especialidad nos impide saber si los alumnos pueden adquirir el nivel suficiente para preparar la prueba de acceso en estos conservatorios. De hecho, la gran cantidad de instrumentos existentes en la especialidad de percusión señalada -caja, batería, instrumentos de láminas, instrumentos de membrana, instrumentos folclóricos y populares- es un indicio de que probablemente dichos estudiantes se verán en clara desventaja a la hora de preparar unas pruebas de acceso frente a los que se hayan preparado en escuelas específicas de jazz.

\subsection{La diversidad de pruebas de acceso}

Como vimos anteriormente, la ley estatal vigente sobre las pruebas de acceso es muy laxa, indicando solamente que en el examen tiene que haber una parte interpretativa, otra de análisis y otra de improvisación. Posteriormente, son las comunidades autónomas las que precisan cómo será cada prueba, y tras esto cada centro puede realizar variaciones en la prueba para adecuarla a su idiosincrasia.

Por todo esto, nos encontramos con pruebas de acceso que a simple vista pueden parecer similares, pero que varían enormemente en varios aspectos, como el porcentaje que vale cada parte en la nota final, la ausencia o no de prueba auditiva y/o de repentización, la obligatoriedad o no de aprender estándares de jazz ${ }^{4}$ de un listado, poder acudir o no con un grupo a la prueba, etc. Todo esto hace que muchos alumnos -e incluso los profesores que les preparan- puedan encontrarse perdidos en un mar de pruebas de acceso.

Además, si asumimos la tesis de que las Enseñanzas Profesionales deberían servir para preparar la prueba de acceso a los Estudios Superiores, deberíamos hacer previamente un estudio profundo de las pruebas de acceso existentes, que hoy en día no existe. Muchos centros se basan principalmente en los requisitos de las pruebas de los conservatorios que llevan impartiendo las Enseñanzas Superiores de Jazz desde principios del presente siglo -especialmente Musikene y Esmuc-, pero la realidad es que casi veinte años después la oferta es mucho más amplia, y las pruebas de acceso varían lo suficiente como para realizar un análisis exhaustivo de ellas.

Un punto a añadir, según Ponce de León (2017) es que en música clásica sigue existiendo un desnivel entre los resultados de aprendizaje adquiridos en el sexto año de Estudios Profesionales y los requisitos exigidos en las pruebas de acceso a los Estudios Superiores. El autor también reivindica una mayor coordinación entre conservatorios profesionales y superiores, así como un diálogo para conseguir un consenso entre los diseñadores de las pruebas y los profesores que programan las asignaturas en los conservatorios profesionales. De esta forma, este estudio pretende ser un primer paso para recopilar las distintas maneras de realizar una prueba de acceso a los Estudios Superiores

\footnotetext{
${ }^{4}$ Traducción de jazz standards, término usual del jazz que hace referencia a las composiciones estándares del repertorio de esta música, conocidas por la mayor parte de los intérpretes.
} 
de Jazz, y así ayudar a que se cree ese diálogo entre los que diseñan dicha prueba y los profesores programadores de las asignaturas de los Estudios Profesionales.

Por todo esto, con este artículo intentaremos averiguar cómo ha de prepararse un alumno para entrar a según qué centro, e intentaremos también servir de ayuda a los encargados de diseñar los Estudios Profesionales, para que puedan diseñar el currículo conociendo la realidad de las distintas pruebas de acceso a los centros superiores. No menos importante, intentaremos abrir el debate de cómo debería realizarse el diseño de una prueba de acceso, tanto en la forma como en el contenido.

\subsection{Antecedentes}

En España existen tres niveles de enseñanzas regladas de música: Enseñanzas Elementales, Profesionales y Superiores. Como hemos mencionado anteriormente, el jazz no comenzó a impartirse en las Enseñanzas Superiores hasta el año 2001, y su equivalente en las Enseñanzas Profesionales se oferta desde hace apenas una década, en una minoría de los conservatorios del país, bajo el nombre de música moderna. Mientras que los centros superiores de jazz ofrecen varias especialidades instrumentales, pudiendo elegir ellos mismos cuáles ofertar, las especialidades impartidas en los conservatorios profesionales son escasas ${ }^{5}$.

Debido a la escasa implementación de las enseñanzas de jazz, y a la escasa formación universitaria del profesorado de estas especialidades, la mayoría de los estudios realizados están enfocados a la música clásica. Sin embargo, existen algunas tesis, artículos y trabajos que hemos tenido en cuenta para realizar este estudio.

Por una parte, existen varias tesis y artículos sobre la enseñanza del jazz en los conservatorios superiores, como las tesis sobre el saxo de Pérez (2012) y Andueza (2017). Por otro lado, SánchezAndrade (2016) habla en su tesis de la importancia de enseñar estilos derivados del jazz en las distintas enseñanzas de percusión, así como la conveniencia de que la batería sea un instrumento de peso dentro de ellas.

Existe también una tesis sobre la prueba de acceso a los Estudios Superiores de clarinete (Garres, 2017), que asegura que el nivel técnico y musical de dichas pruebas supera con creces lo que plantea la legislación para las Enseñanzas Profesionales. Esto nos induce a pensar que, en el caso del jazz, que tiene una institucionalización mucho más joven, pueda estar ocurriendo lo mismo, ya sea en las especialidades existentes en la actualidad -guitarra y bajo eléctrico- o en las que hipotéticamente se puedan crear en el futuro.

Remarcamos también el trabajo de fin de master realizado por Franch (2018), que analiza la enseñanza de la batería en la asignatura de percusión en las Enseñanzas Profesionales, y ofrece distintas posibilidades - dentro del marco legal actual o incluso cambiando leyes- para que los alumnos puedan preparar la prueba de batería de jazz dentro de los conservatorios profesionales. ${ }^{6}$

\footnotetext{
${ }^{5}$ Las especialidades de Estudios Superiores más comunes son batería, bajo eléctrico, contrabajo, guitarra eléctrica, piano, canto, saxofón, trombón y trompeta. Por su parte, los conservatorios que ofrecen Enseñanzas Profesionales de música moderna solo incluyen las especialidades de guitarra eléctrica, bajo eléctrico y percusión moderna (ésta última solamente en Cataluña, desde 2017).

${ }^{6}$ Entre todas las opciones que ofrece, por viabilidad escoge implantar una asignatura optativa de batería jazz para los cursos quinto y sexto de Enseñanzas Profesionales, y desarrolla una programación para esta hipotética optativa. Pese a que podría ser una solución que mejoraría la situación actual,
} 
Otro trabajo de fin de máster (Bernal, 2013) trata la enseñanza del bajo eléctrico en las Enseñanzas Profesionales y Superiores, y muestra los planes de estudio de guitarra eléctrica y bajo eléctrico y sus carencias, desde el punto de vista del autor. Además, el trabajo plantea la cuestión de hasta qué punto están funcionando las Enseñanzas Profesionales para preparar a bajistas para acceder a los Estudios Superiores.

\section{Objetivos}

Conociendo las dificultades que tienen los estudiantes que desean cursar los Estudios Superiores de Jazz, este trabajo se plantea los siguientes objetivos:

1. Identificar las similitudes y diferencias entre los contenidos exigidos en las distintas partes de las pruebas de acceso a los Estudios Superiores de Jazz existentes en España.

2. Averiguar cómo califica cada centro, y qué peso tiene cada parte de la prueba y el expediente del Título Profesional de Música en la nota final.

3. Diseñar una prueba tipo con las características más repetidas en las distintas pruebas de acceso.

4. Realizar una comparación de los datos obtenidos y extraer conclusiones que ayuden a entender el sistema de acceso a los Estudios Superiores de Jazz en España.

5. Plantear líneas de investigación que puedan ayudar a mejorar el sistema de acceso a dichos estudios.

\section{Metodología}

Para este estudio hemos realizado un análisis comparativo de las pruebas de acceso a los Estudios Superiores de Jazz existentes en España hasta la fecha7. Tras ello, hemos extraído diversas conclusiones, por un lado, buscando similitudes entre las distintas pruebas, basándonos en las variables que más se repiten en las tablas. Por otro lado, hemos buscado diferencias entre las distintas pruebas de acceso, resaltando aquellas que nos resultan significativas.

Para realizar el análisis hemos dividido la prueba en tres bloques, que corresponden a las tres partes que señala el Boletín Oficial del Estado (BOE) mencionadas anteriormente: la parte teórica, la parte interpretativa y la parte de improvisación. En esta última hemos incluido la repentización, ya que, aunque no sea mencionada por el BOE, aparece en todas las pruebas de acceso, y suele realizarse al mismo tiempo que la prueba de improvisación, o estar mezclada con ella.

Para adquirir esta información hemos accedido a la normativa de cada comunidad autónoma, que en muchos casos define cómo ha de ser la prueba, y posteriormente hemos rastreado la información de las pruebas de acceso existente en cada centro. En algunos casos, donde la información era confusa, hemos contactado con los profesores de batería de los centros.

\footnotetext{
entendemos que, de todas las opciones posibles que da el autor, la ideal sería crear la especialidad de batería jazz durante toda la Enseñanza Profesional, ya que consideramos que dos años son insuficientes para preparar unas pruebas de acceso.

${ }^{7}$ Queda fuera de este análisis el Conservatorio Superior de Música de Canarias, ya que el itinerario jazz comienza en tercer curso de Superior para la mayoría de los instrumentos, por lo que la prueba de acceso es para música clásica.
} 
Una vez obtenida toda la información, hemos realizado una tabla comparativa con las siguientes características.

\subsection{Valor de cada parte de la prueba en la nota final}

Para calcular el valor que aporta cada parte a la nota final de la prueba hemos tenido que recalcular los porcentajes que ofrecía la normativa o la web de cada centro. En la gran parte de los casos las pruebas no estaban divididas exactamente en teórica, interpretativa y repentización/improvisación; por lo que hemos tenido que recalcular dichos porcentajes en varios $\operatorname{casos}^{8}$.

Hay un último dato que cada comunidad autónoma valora de una forma distinta: se trata de la nota que obtuvo el candidato en el expediente de las Enseñanzas Profesionales en caso de haberlas cursado. Debido a la gran diferencia de criterios $^{9}$ hemos decidido incluir este valor aparte, de manera que se simplifique la lectura de los porcentajes de cada una de las partes mencionadas anteriormente.

\subsection{Descripción de cada tabla}

También hemos realizado tablas en las que se pueda observar de manera sintética en qué consiste cada una de las partes, para poder comparar visualmente unas pruebas con otras.

La parte teórica tiene tres secciones, que no tienen por qué estar estructuradas de esa manera en cada examen, ya que varían según el centro. Dicha parte la componen la sección de análisis melódico, armónico, estructural/formal, instrumental, textura/arreglos-, la sección auditiva dictado melódico, dictado armónico, intervalos- y una tercera sección a la que hemos llamado "otros" -escribir o averiguar escalas, acordes e intervalos; y preguntas sobre cultura e historia del jazz-. Esta tabla tiene dos únicos valores posibles, sí o no, para facilitar el análisis comparativo.

La tabla para la parte interpretativa tiene a su vez cuatro secciones: interpretación de un programa, interpretación de estándares de jazz de un listado, interpretación de estudio técnico, y sesión de trabajo en la que los candidatos dan instrucciones a otros músicos y reciben instrucciones del tribunal. En esta tabla indicamos la duración o el número de temas establecidos en "programa" y "estándares", y valores "sí" o "no" en el caso de que existan o no el estudio técnico y/o la sesión de trabajo.

Para la tabla de repentización e improvisación nos hemos basado en la prueba de batería, ya que dichas partes varían en función del tipo de instrumento -melódico, armónico o rítmico-. En esta parte podemos encontrarnos con lectura tipo big band ${ }^{10}$, imitación de oído de ciertos patrones e improvisación ${ }^{11}$.

\footnotetext{
${ }^{8}$ Los porcentajes se han redondeado al número entero más próximo para facilitar la interpretación de los datos.

${ }^{9}$ En algunas comunidades autónomas no tener el Título Profesional puede bajar la nota drásticamente, en otras se valora en un porcentaje muy bajo y dan la opción de hacer un examen a quien no lo tenga, etc.

${ }^{10}$ Lectura en la que aparecen kicks -golpes con indicaciones de articulación sin indicar en qué instrumento se tocarán- que el baterista interpretará como crea conveniente. También aparecen fills -partes no escritas que el baterista deberá rellenar con golpes elegidos por él.

${ }^{11}$ Esta puede ser sobre una partitura, una improvisación libre, o sobre una secuencia interpretada por músicos o reproducida por un play-along.
} 


\section{Resultados}

En primer lugar, mostramos el porcentaje que vale cada parte de la prueba de acceso a los Estudios Superiores de Jazz. Para simplificar la lectura hemos indicado la provincia o ciudad de cada centro, y en los casos en los que haya más de un centro por provincia, hemos utilizado el nombre por el que se conoce popularmente a cada institución. Sevilla y Málaga, aunque sean centros distintos, se incluyen juntos porque los datos de las variables analizadas para las pruebas son idénticos ${ }^{12}$.

En la Tabla 1, las columnas que corresponden a las tres partes mencionadas de la prueba -teórica, interpretativa, y repentización e interpretación-suman entre ellas $100 \%$. A la derecha mostramos el valor del expediente de los Estudios Profesionales.

Tabla 1

Porcentaje que vale cada parte de la prueba de acceso y el expediente del Título Profesional

\begin{tabular}{ccccc} 
Centro & Teórica & Interpretativa & $\begin{array}{c}\text { Repentización, } \\
\text { improvisación }\end{array}$ & $\begin{array}{c}\text { Expediente } \\
\text { EEPP }\end{array}$ \\
\hline UAX & & & 20 & 30 \\
Creativa & 20 & 60 & 12 & 30 \\
Mayeusis & 20 & 68 & 8 & 40 exc. \\
A Coruña & 25 & 67 & 8 & 40 exc. \\
Valga & 25 & 67 & 8 & 40 exc. \\
San Sebastián & 25 & 67 & 40 & $+0,5 /+1,2$ \\
Pamplona & 20 & 40 & 35 & $5(+0,5)$ \\
Esmuc & 25 & 40 & 15 & $5(+0,5)$ \\
Liceu & 35 & 50 & 15 & $5(+0,5)$ \\
Taller de Músics & 35 & 50 & 20 & $5(+0,5)$ \\
Jam Session & 35 & 50 & 33 & 50 \\
Islas Baleares & 35 & 45 & 19 & 50 \\
Valencia & 33 & 33 & 40 & 40 \\
Sevilla y Málaga & 36 & 45 & 40 & 8 \\
\hline
\end{tabular}

Nota. UAX: Universidad Alfonso X el Sabio de Madrid. Creativa: Escuela de Música Creativa de Madrid. Fuente: elaboración propia basada en la información obtenida de las páginas web de cada conservatorio mencionado en la tabla.

La aplicación de la nota del expediente de los Estudios Profesionales varía según la comunidad autónoma. En la mayor parte de los casos, y siempre que el alumno haya cursado dichos estudios, habrá que calcular la nota final sumando el porcentaje indicado del expediente al porcentaje asignado a la prueba de acceso ${ }^{13}$. En caso de no haberlos cursado, la nota de la prueba valdrá el $100 \%$.

En los centros catalanes la prueba de acceso valdrá un $95 \%$, y el $5 \%$ restante valdrá un 10 para los que tengan el Título Profesional, independientemente de su nota en el expediente. Para los que no lo tengan, habrá un examen especial de conocimientos musicales, y la nota obtenida se enmarcará en este $5 \%$. En el caso de Navarra, la nota obtenida en la prueba valdrá el $100 \%$, a la que se sumará entre 0,5 y 1,2 puntos en función de la nota obtenida en el expediente de los Estudios Profesionales. Finalmente, en el caso de Galicia el $40 \%$ será excluyente, es decir, si el estudiante no ha realizado los Estudios Profesionales, obtendrá cero puntos en esta parte.

\footnotetext{
${ }^{12}$ Para las conclusiones, sin embargo, se tratarán como conservatorios distintos.

${ }^{13}$ Es el caso de los centros de Madrid, País Vasco, Islas Baleares, Comunidad Valenciana y Andalucía. Si el expediente vale un $30 \%$, la nota obtenida en la prueba de acceso valdrá el restante hasta llegar a $100 \%$, en este caso un $70 \%$.
} 
Para la parte de la prueba teórica hemos realizado dos tablas. En la Tabla 2 agrupamos las características de la prueba de análisis escrito.

Tabla 2

Prueba teórica: características de la prueba de análisis escrito en cada centro

\begin{tabular}{|c|c|c|c|c|c|}
\hline Centro & Melódico & Armónico & $\begin{array}{c}\text { Estructural y } \\
\text { formal }\end{array}$ & Instrumental & $\begin{array}{r}\text { Textura y } \\
\text { arreglos }\end{array}$ \\
\hline UAX & & Sí & & & \\
\hline Creativa & & Sí & Sí & Sí & Sí \\
\hline Mayeusis & Sí & Sí & Sí & & \\
\hline A Coruña & Sí & Sí & Sí & & \\
\hline Valga & Sí & Sí & Sí & & \\
\hline San Sebastián & & Sí & & & \\
\hline Pamplona & & Sí & Sí & & \\
\hline Esmuc & Sí & Sí & Sí & & Sí \\
\hline Liceu & Sí & Sí & Sí & & \\
\hline Taller de Músics & & Sí & Sí & Sí & Sí \\
\hline Jam Session & & Sí & Sí & Sí & \\
\hline Islas Baleares & Sí & Sí & Sí & Sí & \\
\hline Valencia & Sí & Sí & Sí & & \\
\hline Sevilla y Málaga & Sí & Sí & Sí & & \\
\hline
\end{tabular}

Nota. Los espacios en blanco significan "No". Fuente: elaboración propia basada en la información obtenida de las páginas web de cada conservatorio mencionado en la tabla.

En la Tabla 3, también sobre la parte teórica, hemos recopilado cómo es la prueba auditiva en cada centro.

Tabla 3

Prueba teórica: características de la prueba auditiva en cada centro

\begin{tabular}{|c|c|c|c|}
\hline Centro & Dictado melódico & Dictado armónico & Intervalos \\
\hline \multicolumn{4}{|l|}{ UAX } \\
\hline Creativa & Sí & Sí & Sí \\
\hline Mayeusis & & Sí & \\
\hline A Coruña & & Sí & \\
\hline Valga & & Sí & \\
\hline San Sebastián & Sí & Sí & \\
\hline \multicolumn{4}{|l|}{ Pamplona } \\
\hline Esmuc & Sí & Sí & \\
\hline Liceu & Sí & Sí & \\
\hline Taller de Músics & Sí & Sí & \\
\hline Jam Session & Sí & Sí & \\
\hline Islas Baleares & Sí & Sí & \\
\hline \multicolumn{4}{|l|}{ Valencia } \\
\hline Sevilla y Málaga & & & \\
\hline
\end{tabular}

Nota. Los espacios en blanco significan "No". Fuente: elaboración propia basada en la información obtenida de las páginas web de cada conservatorio mencionado en la tabla.

La Tabla 4 muestra otro tipo de pruebas que puedan aparecer en la parte teórica de algunos centros. Estas pruebas son siempre escritas, pudiendo ser de reconocimiento -reconocer escalas, intervalos o acordeso de desarrollo -escribir la escala, intervalo o acorde indicado, o bien responder a preguntas teóricas-. 
Tabla 4

Prueba teórica: existencia o no de otras pruebas escritas en cada centro

\begin{tabular}{|c|c|c|c|}
\hline Centro & Escalas & Intervalos & Acordes \\
\hline \multicolumn{4}{|l|}{ UAX } \\
\hline Creativa & Sí & & Sí \\
\hline \multicolumn{4}{|l|}{ Mayeusis } \\
\hline \multicolumn{4}{|l|}{ A Coruña } \\
\hline \multicolumn{4}{|l|}{ Valga } \\
\hline San Sebastián & & Sí & Sí \\
\hline Pamplona & Sí & & \\
\hline \multicolumn{4}{|l|}{ Esmuc } \\
\hline Liceu & Sí & & \\
\hline Taller de Músics & & & Sí \\
\hline \multicolumn{4}{|l|}{ Jam Session } \\
\hline Islas Baleares & Sí & & \\
\hline Valencia & & Sí & Sí \\
\hline Sevilla y Málaga & & & \\
\hline
\end{tabular}

Nota. Los espacios en blanco significan "No". Fuente: elaboración propia basada en la información obtenida de las páginas web de cada conservatorio mencionado en la tabla.

En cuanto a la parte interpretativa, hemos recogido la información en una sola tabla. La parte "programa" indica la sección de la prueba que puede preparar el candidato días antes del examen, que se suele interpretar con músicos que este aporte o con play-along. Esta parte puede ser totalmente libre, estar sujeta a un repertorio concreto, o basada en ciertos estándares de jazz o épocas. En la Tabla 5 se indica la duración de este repertorio en cada centro, que suele venir dada en tiempo -mínimo o aproximado-y/o en número de temas.

Tabla 5

Prueba teórica: existencia o no de otras pruebas escritas en cada centro

\begin{tabular}{|c|c|c|c|c|}
\hline Centro & Programa & Estándar & $\begin{array}{l}\text { Estudio } \\
\text { técnico }\end{array}$ & $\begin{array}{c}\text { Sesión de } \\
\text { trabajo }\end{array}$ \\
\hline UAX & $>15^{\prime}$ & & Sí & \\
\hline Creativa & 20 a $30^{\prime}$ & & Sí & \\
\hline Mayeusis & $15^{\prime}$ & $15^{\prime}$ & & \\
\hline A Coruña & $15^{\prime}$ & $15^{\prime}$ & & \\
\hline Valga & 5 temas & & & \\
\hline San Sebastián & $10^{\prime}$ & $15^{\prime}$ & & Sí \\
\hline Pamplona & $10^{\prime}, 2$ temas & 1 tema & & \\
\hline Esmuc & $>30^{\prime}, 4$ temas & & Sí & Sí \\
\hline Liceu & 4 temas & & Sí & Sí \\
\hline Taller de Músics & $>30^{\prime}, 4$ temas & & Sí & Sí \\
\hline Jam Session & 5 temas & & Sí & Sí \\
\hline Islas Baleares & $>15^{\prime}, 4$ temas & & & \\
\hline Valencia & & 1 tema & Sí & \\
\hline Sevilla y Málaga & Sí & Sí & Sí & \\
\hline
\end{tabular}

Nota. Los espacios en blanco significan "No". Fuente: elaboración propia basada en la información obtenida de las páginas web de cada conservatorio mencionado en la tabla. 
En la misma tabla vemos la columna "estándar", que indica que cada aspirante tendrá que interpretar uno o varios estándares de jazz elegidos entre varios ofrecidos el día de la prueba, que a su vez pertenecen a un listado ofrecido previamente por el centro ${ }^{14}$.

El "estudio técnico", por su parte, consiste en un solo de caja o transcripción de solo de batería que deberá elegirse libremente o de un listado ofrecido por el centro ${ }^{15}$. La última columna, "sesión de trabajo", corresponde a un ejercicio en el que cada aspirante debe ejercer de líder de grupo, dando instrucciones al resto de músicos que le acompañarán; y, en algunos casos, responder a instrucciones dadas por el tribunal.

Por último, recopilamos las características de la prueba de repentización e improvisación en la Tabla 6. Por una parte, podemos ver los centros que exigen una lectura a primera vista, con kicks en el caso de la batería -partitura tipo big band-. Otros centros piden imitar patrones a oído e improvisar sobre ellos; $y$ otros una improvisación sobre una base, con otros músicos, con partitura, o libre -o combinaciones entre estas-.

Tabla 6

Prueba de repentización e improvisación: características de cada centro

\begin{tabular}{|c|c|c|c|}
\hline Centro & Kicks, big band & Imitación de patrones & $\begin{array}{c}\text { Improvisación: Base (B) / } \\
\text { Músicos (M)/ Partitura (P) } \\
\text { / Libre (L) }\end{array}$ \\
\hline UAX & Sí & & \\
\hline Creativa & Sí & & \\
\hline Mayeusis & Sí & & Sí (M/P) \\
\hline A Coruña & Sí & & Sí (P) \\
\hline Valga & Sí & & Sí (P) \\
\hline San Sebastián & Sí (I) & Sí (I) & \\
\hline Pamplona & Sí (I) & & \\
\hline Esmuc & Sí & & Sí (B/P) \\
\hline Liceu & & & Sí \\
\hline Taller de Músics & & & Sí (B/P) \\
\hline Jam Session & Sí (rock) & & Sí (B/L) \\
\hline Islas Baleares & Sí & & Sí (B) \\
\hline Valencia & Sí & Sí (I) & Sí (P) \\
\hline Sevilla y Málaga & Sí & & \\
\hline
\end{tabular}

Nota. Los espacios en blanco significan "No". La letra I entre paréntesis (I) indica que el ejercicio requiere también improvisación. Fuente: elaboración propia basada en la información obtenida de las páginas web de cada conservatorio mencionado en la tabla.

\subsection{Análisis de los resultados}

Una vez analizadas las distintas tablas, extraeremos algunos datos que nos permitan llegar más adelante a algunas conclusiones sobre las distintas pruebas de acceso a los Estudios Superiores de Jazz que se realizan en España.

\subsubsection{Análisis sobre el valor de cada parte}

En primer lugar, vemos que el porcentaje de la parte dedicada a la interpretación de repertorio es el más alto, entre el 40 \% y el 68 \%, excepto en Baleares (33\%). La parte teórica

\footnotetext{
${ }^{14}$ Excepto en el caso de San Sebastián, que habla de "un estándar de jazz elegido por Musikene", por lo que podrá aparecer cualquier estándar del repertorio jazzístico.

${ }^{15}$ Son muy comunes los solos de Charley Wilcoxon (caja) o las transcripciones de solos de Philly Joe Jones (batería).
} 
varía entre el $20 \%$ y el $36 \%$ y y la de repentización e improvisación entre el $8 \%$ y el $40 \%$. Esta última parte aporta el $15 \%$ o menos en la mitad de los casos.

Por otra parte, comprobamos que poseer el Título Profesional puede ayudar en gran medida a conseguir buena nota en las pruebas de acceso. En la mayor parte de las comunidades autónomas tenerlo vale entre el $30 \%$ y el $50 \%$ de la nota final, correspondiendo este último porcentaje a la Comunidad Valenciana y las Islas Baleares. En Galicia, además, perjudica gravemente no tener dicho título: los que se presenten sin haber cursado las Enseñanzas Profesionales perderán automáticamente el $40 \%$ de la nota. En Cataluña dicho título se valora solo en un $5 \%$, aun siendo esta la comunidad que tiene más trayectoria en las Enseñanzas Profesionales con itinerario jazz e instrumentos modernos. Otras comunidades donde el título se valora menos que en la media son el País Vasco y Navarra.

\subsubsection{Análisis sobre la parte teórica}

Sobre la parte teórica podemos observar que el análisis armónico está presente en todas las pruebas. El análisis melódico, por un lado, y el estructural y formal, por otro; son prácticamente universales. Sin embargo, el análisis instrumental, de la textura o del arreglo aparecen en pocos centros.

De la misma manera, los dictados armónicos son comunes a casi todas las pruebas, y el dictado melódico aparece en gran parte de ellas. Reconocer intervalos de manera auditiva, en cambio, solo es exigido en la Escuela de Música Creativa.

Otras pruebas que pueden aparecer son el reconocimiento de escalas, intervalos o acordes escritos; o bien escribir las notas de una escala, intervalo o acorde dado. Sin embargo, esto se da en apenas una cuarta parte de los centros. Por último, es común a casi todos los centros encontrar preguntas sobre la historia del jazz, versiones de un estándar, músicos del jazz, etc.

\subsubsection{Análisis sobre la parte interpretativa}

Salvo en Valencia, en todos los centros se exige preparar un programa para la prueba, que durará más de diez minutos, y que en algunos casos deberá extenderse más allá de los treinta. Algunos centros piden un número determinado de temas, que suelen ser cuatro o cinco -dos en el caso de Pamplona-.

Por otra parte, casi en la mitad de los casos se pide que los aspirantes preparen algunos estándares de jazz. Los centros que exigen esta prueba incluyen listados en sus páginas web con los estándares que se interpretarán en el examen.

Por último, algo más de la mitad de los centros exigen que se prepare un estudio técnico, y la tercera parte de ellos realizarán una sesión de trabajo en sus pruebas, donde los aspirantes deberán dar instrucciones a los músicos que los acompañen y responder a instrucciones del tribunal. 


\subsubsection{Análisis sobre la parte de repentización e improvisación}

La parte de repentización e improvisación es la que más difiere entre los distintos centros. Aun así, la mayoría de ellos exigen que se interprete a primera vista una partitura con kicks o tipo big band, en la que el baterista tendrá que interpretar ciertos golpes y rellenar con fills como crea conveniente; e incluso improvisar más adelante sobre el mismo fragmento.

La improvisación es imprescindible en el $60 \%$ de los casos, si bien es cierto que se puede presentar al aspirante de diversas maneras. Esta improvisación será, en muchos de los centros, con una partitura y/o con una base grabada; y en dos de los centros se pide que sea libre o con músicos.

\section{Discusión}

Tras haber analizado todos estos datos, extraeremos algunas conclusiones. En primer lugar, describiremos una prueba tipo con las características que aparezcan reiterativamente en cada prueba, que podrá ser útil para estudiantes que todavía no tengan claro a qué centro querrían presentarse, o a pedagogos o diseñadores de currículos de jazz previos a las Enseñanzas Superiores. Tras esto, llegaremos a algunas conclusiones generales sobre las pruebas de acceso.

\subsection{Conclusiones sobre la prueba de acceso tipo}

Como ya hemos comentado, la prueba de acceso consta de tres partes: teórica, interpretativa, y repentización e improvisación. Describimos, a continuación, cómo sería una prueba de acceso tipo, basándonos en las características que se dan en un $60 \%$ o más de los casos.

Por un lado, la parte teórica estaría compuesta por un análisis melódico, armónico, y estructural/formal; y por una sección auditiva con un dictado armónico y un dictado melódico.

Por otro lado, la prueba interpretativa consistiría en la interpretación de un programa de cuatro o cinco temas, con una duración aproximada de treinta minutos, con lo que la duración media de los temas sería de entre seis y siete minutos ${ }^{16}$. El aspirante elegiría entre preparar un repertorio libre, escoger entre el repertorio clásico de estándares de jazz, o hacer una mezcla entre ambos. Varios centros ofrecen listados con estándares de jazz para orientar en este sentido a los aspirantes.

También en la parte interpretativa se exigiría realizar un estudio técnico ofrecido por los centros meses antes de la prueba. En esta parte es común encontrar solos de caja tipo Charley Wilcoxon o transcripciones de solos de batería de Philly Joe Jones.

En la parte de repentización e improvisación se exigiría una lectura tipo kicks y/o big band, basada en interpretar kicks con bombo y caja sin modificar el patrón de ride; y además, una prueba consistente en interpretar kicks con anticipaciones y rellenos (fills), incluyendo también improvisaciones cortas.

\footnotetext{
${ }^{16}$ Dado a las diferentes formas de describir esta prueba por parte de los centros, se ha buscado una definición que cuadre con la mayor parte de ellos, en este caso con un $66 \%$ de las pruebas.
} 


\subsection{El valor de la nota final}

Realizando una media sobre las partes anteriores, la parte teórica valdría el $27 \%$ de la nota final, la parte interpretativa el $51 \%$ y y la parte de repentización e improvisación el $22 \%$. Los porcentajes varían en función del centro, pero la parte interpretativa casi siempre es la más importante. Cabe destacar que hay lugares donde la repentización y la improvisación apenas puntúa, especialmente en los conservatorios gallegos $-8 \%$ de la nota final-; y sin embargo en otros centros esta parte es muy importante $-40 \%$ en los centros andaluces y en Musikene-.

Por otro lado, tener el Título Superior puede ayudar a obtener una mejor puntuación en la prueba, especialmente si se han obtenido altas calificaciones en el expediente. Las comunidades autónomas que más lo valoran son, en este orden, Galicia, Islas Baleares, Comunidad Valenciana y Andalucía ${ }^{17}$.

\subsection{Conclusiones generales}

Como hemos visto a lo largo del artículo, es necesario un debate para averiguar cómo podría el estudiantado prepararse para la prueba de acceso a los Estudios Superiores de Jazz en el sistema público de enseñanza. Todo apunta a que es necesario crear especialidades que se puedan cursar durante todos los cursos de los Estudios Profesionales -batería jazz, trompeta jazz, etc.-, que incluyan asignaturas que les ayuden a preparse para todas las partes de la prueba -armonía y auditiva modernas, combo, big band, etc.

Es necesario también plantearse cuánto debería valer el expediente de las Enseñanzas Profesionales para la nota final prueba de acceso, e intentar entender por qué en Cataluña, la comunidad autónoma con más trayectoria y número de especialidades en los Estudios Profesionales de Jazz, se valora el expediente de dichos estudios en solo un $5 \%$, mientras que en otras comunidades sin dicha especialidad el expediente se valora hasta un $50 \%$. Habría que preguntarse, del mismo modo, si es adecuado o no penalizar a los que no poseen dicho título como ocurre en Galicia.

La parte interpretativa siempre está más valorada que la teórica y la de repentización e improvisación ${ }^{18}$, por lo que el conjunto de aspirantes debería tener siempre en cuenta el gran peso de esta parte. Dicho esto, no debería menospreciarse la parte teórica, ya que en casi todos los casos supone el $25 \%$ de la prueba o más.

La parte de repentización e improvisación podría influir en los estudiantes a la hora de decidir a qué centro presentarse. Aquellos que consideren que tienen buenas habilidades de lectura a primera vista e improvisación pueden partir con cierta ventaja para entrar en San Sebastián, Andalucía, Pamplona o Islas Baleares; mientras que los que no tengan tan trabajados estos aspectos pueden tener más facilidades para ser admitidos en los conservatorios de Galicia -siempre que hayan terminado los Estudios Profesionales-, Cataluña, Valencia o Madrid.

\subsection{Limitaciones}

Este estudio ha tenido ciertas limitaciones, ya que se ha basado prácticamente solo en el análisis de la normativa y de la información disponible en internet sobre las pruebas de acceso a los Estudios

\footnotetext{
${ }^{17}$ Galicia aparece en primer lugar al ser la única comunidad autónoma que penaliza a los estudiantes que no poseen el Título Profesional.

${ }^{18}$ Salvo en Islas Baleares, donde todas las partes valen lo mismo.
} 
Superiores de Jazz de los centros de todo el país, y que se relacionan con las posibles vías de investigación que veremos en el siguiente epígrafe. De este modo, no hemos podido analizar cómo son en la práctica las pruebas de acceso en cada centro, y si cumplen estrictamente con el desarrollo de la prueba explicado en sus respectivas páginas web.

Este estudio tampoco ha analizado en profundidad si los centros ofrecen músicos a los aspirantes, si estos tienen que acudir con su propia banda, si pueden utilizar o no play-alongs... etc. Por limitaciones de extensión, tampoco hemos realizado un análisis sobre los estándares de jazz que se piden en los listados de cada centro.

Otras variables que no se han tenido en cuenta para este estudio son la mayor relación oferta/demanda -como norma general- entre los centros privados y los públicos o concertados, o la alta demanda en los conservatorios de San Sebastián y Barcelona.

Por último, tampoco hemos analizado otras variables que pueden influir en las posibilidades de cada aspirante, como la existencia del miedo escénico, la forma de trabajar la preparación de la prueba, el estudio del día a día, las cuestiones psicológicas, etc.

\subsection{Prospectiva}

El presente artículo puede ser un primer paso hacia investigaciones futuras que permitan la mejora de la enseñanza del jazz en España, especialmente durante las Enseñanzas Profesionales y en el paso a los Estudios Superiores.

Una posible nueva vía de investigación podría consistir en realizar un estudio cualitativo in situ sobre cómo son las pruebas de acceso, realizando entrevistas a diferentes aspirantes y analizando distintas situaciones que se puedan dar en la prueba. Algunas de estas situaciones a analizar podrían ser la posibilidad o no de afinar el instrumento antes de comenzar a tocar, de llevar material como platillos y caja, de disponer de más o menos minutos para preparar la repentización, las instrucciones que se dan exactamente en la sesión de trabajo, o el tempo al que se deben realizar los ejercicios de repentización.

Sería interesante también establecer un diálogo en cuanto al formato de prueba de acceso más adecuado, ya que muchos centros ofrecen la posibilidad de acudir a la prueba de acceso con una banda propia, lo que puede ayudar al aspirante; pero que a su vez puede generar desigualdades, especialmente para aquellos que se presenten en comunidades autónomas lejos de su lugar de residencia, por las dificultades que conlleva llevar a los propios músicos.

Otra vía de investigación que podría completar este estudio sería realizar un análisis sobre cuál sería el repertorio de estándares de jazz adecuado para acceder a los Estudios Superiores de Jazz. En este caso, sería interesante realizar un consenso a nivel estatal que pudiese establecer un repertorio fijo de estándares, para aspirantes que quieran presentarse a más de un centro, que además pudiese homogeneizar las pruebas de acceso y facilitar el diseño de currículos de las Enseñanzas Profesionales.

Más allá de estos puntos concretos, otra futura línea de investigación sería analizar el papel que debería tener el jazz en las Enseñanzas Profesionales de música, y si habría que ampliarlo más allá de los perfiles actuales que ocupan los dos últimos cursos, o si habría que crear especialidades 
equivalentes a las actuales de bajo eléctrico y guitarra eléctrica, e incluso si habría que mejorar estas con asignaturas más enfocadas al jazz que permitiesen preparar la prueba de acceso a los Estudios Superiores.

Por último, sería conveniente crear una propuesta metodológica para batería jazz e implantarla en los conservatorios profesionales que ofrezcan estos itinerarios, analizando los resultados. La mayor parte de los estudiantes siguen preparando las pruebas de acceso con profesores particulares o en clases privadas, por lo que la posibilidad de preparar las pruebas de acceso dentro del sistema público garantizaría la igualdad y el acceso a alumnos que, por ciertas condiciones -generalmente económicas o geográficas- tienen más dificultades para acceder a los Estudios Superiores de Jazz.

\section{Organismos colaboradores}

Universidad Politécnica de Madrid, Real Conservatorio Superior de Música de Madrid.

\section{Referencias}

Andueza, M. (2017). Análisis de la situación de la enseñanza del Saxo Jazz en la Comunidad Autónoma Vasca y Navarra [Tesis doctoral, Universidad del País Vasco].

Bernal, A. (2013). 10 Años de Enseñanza Formal del Bajo Eléctrico en España [Trabajo de Fin de Máster, Universidad Pública de Navarra].

Calderón, D. (2016). El jazz y la música moderna en los Conservatorios Superiores: una inclusión a tempo Adagio. En A. M. Vernia Carrasco (Ed.), Las enseñanzas superiores de música en el territorio español. Diferentes situaciones y contextos (pp. 93-101). Siníndice.

Casas, A. (2013). Culturas de aprendizaje musical: concepciones, procesos y prácticas de aprendizaje en Clásico, Flamenco y Jazz [Tesis doctoral, Universidad Autónoma de Madrid].

Decreto 5/2017, de 17 de enero, de modificación del Decreto 25/2008, de 29 de enero, por el que se establece la ordenación curricular de las enseñanzas de música de grado profesional y se regula la prueba de acceso, para incluir varias especialidades y po. (s. f.). Diari Oficial de la Generalitat de Catalunya, núm. 7291, de 20 de enero de 2017.

Franch, R. (2018). La batería en las Enseñanzas Profesionales de música [Trabajo de Fin de Máster, Universitat Jaume I].

Garres, F. J. (2017). La prueba de acceso a grado superior de Clarinete como eje vertebrador de la docencia en el último ciclo de Enseñanzas Profesionales: propuesta metodológica [Tesis doctoral, Universidad Católica de Murcia].

Ley Orgánica 1/1990, de 3 de octubre, de Ordenación General del Sistema Educativo (LOGSE). (s. f.). Boletín Oficial del Estado, núm 238, de 4 de octubre de 1990.

Pérez, V. (2012). Propuesta de un método teórico-práctico para desarrollar la técnica de base común en las especialidades de Clásico, Moderno y Jazz para su integración en el sistema educativo actual [Tesis doctoral, Universidad Politécnica de Valencia]. https://doi.org/10.4995/thesis/10251/21569 
Ponce de León, L. (2017). Las Enseñanzas Profesionales de Música: Análisis Dafo. Música Oral del Sur, 14, 253-260.

Real Decreto 617/1995, de 21 de abril, por el que se establece los aspectos básicos del currículo del grado superior de las enseñanzas de Música y se regula la prueba de acceso a estos estudios. (s. f.). Boletín Oficial del Estado, núm. 134, de 6 de junio de 1995.

Real Decreto 756/1992, de 26 de junio, por el que se establece los aspectos básicos del currículo de los grados elemental y medio de las enseñanzas de música. (s. f.). Boletín Oficial del Estado, núm. 206, de 27 de agosto de 1992.

Sánchez-Andrade, J. M. (2016). La pedagogía de la Percusión en el grado superior de los conservatorios españoles: el patrimonio como referencia para innovar con equidad [Tesis doctoral, Universidad de Oviedo]. 